\title{
Review Article Tropical Infections in the Context of Kidney Transplantation in Latin America
}

\author{
Lúcio R. Requião-Moura, ${ }^{1,2 *}$ Elizabeth De Francesco Daher, ${ }^{3}$ Cassio R. Moreira Albino, ${ }^{1}$ Savio de Oliveira Brilhante, ${ }^{3}$ \\ Geraldo Bezerra da Silva Junior, ${ }^{4}$ Silvana Daher Costa, ${ }^{3,5}$ and Tainá Veras de Sandes-Freitas ${ }^{3,5}$ \\ ${ }^{1}$ Renal Transplant Unit, Hospital Israelita Albert Einstein, São Paulo, Brazil; ${ }^{2}$ Nephrology Division, Federal University of São Paulo, São Paulo, Brazil; \\ ${ }^{3}$ Department of Clinical Medicine, Federal University of Ceará, Fortaleza, Brazil; ${ }^{4}$ Medical School, University of Fortaleza, Fortaleza, Ceará, Brazil; \\ ${ }^{5}$ Hospital Geral de Fortaleza, Fortaleza, Ceará, Brazil
}

\begin{abstract}
Reports on tropical infections among kidney transplant (KT) recipients have increased in recent years, mainly because of the growing number of KT programs located in tropical and subtropical areas, and greater mobility or migration between different areas of the world. Endemic in emerging and developing regions, like most countries in Latin America, tropical infections are an important cause of morbidity and mortality in this population. Tropical infections in KT recipients may exhibit different pathways for acquisition compared with those in nonrecipients, such as transmission through a graft and reactivation of a latent infection triggered by immunosuppression. Clinical presentation may differ compared with that in immunocompetent patients, and there are also particularities in diagnostic aspects, treatment, and prognosis. KT patients must be screened for latent infections and immunized properly. Last, drug-drug interactions between immunosuppressive agents and drugs used to treat tropical infections are an additional challenge in KT patients. In this review, we summarize the management of tropical infections in KT patients.
\end{abstract}

\section{INTRODUCTION}

Infections are the main cause of death in kidney transplant (KT) recipients living in emerging and developing countries. ${ }^{1-3}$ Even in developed countries, where cardiovascular events are the main cause of death, infections are among the three leading causes of mortality. ${ }^{4}$ The increasing number of published reports on tropical infections in KT patients is remarkable. The growing number of transplant programs in emerging and developing countries in tropical and subtropical areas such as Brazil, Argentina, Mexico, India, Africa, and China partially justifies this higher incidence. ${ }^{5}$

In addition, improvement in patients' life expectancy and better quality of life results in greater mobility or migration between different areas in the world, with consequently greater exposure to environmental risks. Last, transplant tourism also contributes to this scenario. ${ }^{6,7}$ Most tropical infections are considered neglected diseases by the WHO and occur mainly in countries with fragile health systems, resulting in inadequate surveillance strategies and low notification rates. Given this big picture, the actual incidence of tropical infections in these countries must be greater.

In this setting, a better understanding of the interactions between tropical infections and KT has a pivotal role in improving health care and patient outcomes. This review aimed to address the peculiarities of the occurrence of tropical infections in Latin America in the context of kidney transplantation.

\section{PATHWAYS OF TRANSMISSION}

The transmission of tropical infections in the KT scenario may occur via three pathways: 1) de novo infection, 2) reactivation, or 3) donor-derived infection. De novo infection is defined as the direct acquisition of infection from the community of transplanted patients who had never been in contact with the pathogen before, or the re-exposure to the same

*Address correspondence to Lúcio R. Requião-Moura, Rua Botucatu, 591, $15^{\circ}$ Andar, Vila Clementino, São Paulo, Brazil, 04023-062. E-mail: lucio.moura@einstein.br pathogen in previously exposed and properly treated patients. This pathway is more common after the first year after KT, when patients are fully recovered and engaged in their regular activities, such as jobs and social life. Similar to the general population, it occurs frequently among people living in or visiting tropical areas. ${ }^{5,6}$

Another mechanism is reactivation or recrudescence of latent diseases, such as tuberculosis (TB), severe forms of strongyloidiasis, Chagas disease, leishmaniasis, and malaria. This phenomenon is more frequent during the first 6 months after KT and is a result of the intense immunosuppressive state that occurs during the early posttransplant period. ${ }^{5,6}$ Less commonly, tropical infections can be acquired by transmission from the donor through the allograft. Clinical presentation is usually early, but may occur at any time after KT. Chagas disease, TB, malaria, and human T-cell leukemia virus type 1 (HTLV-1) are examples of reported donor-derived infections. ${ }^{5,8,9}$

\section{PECULIARITIES OF CLINICAL PRESENTATION AND TREATMENT}

The diagnosis is sometimes more difficult in KT patients than in immunocompetent patients, because the immune response is modified by immunosuppressive drugs, resulting in atypical presentation. ${ }^{10}$ Common findings, such as eosinophilia in strongyloidiasis, granulomas in TB and schistosomiasis, cyclical fever in malaria, and organomegalies in visceral leishmaniasis, can be absent. ${ }^{11-15}$ Furthermore, malaria, Chagas disease, and strongyloidiasis may exhibit a more aggressive and catastrophic evolution in KT patients, with a high risk for severe organ damage and death.,12 Although some evidence has suggested that KT patients are less likely to develop severe dengue, depending on intense T-lymphocyte activity, ${ }^{6,9}$ a recently published systematic review reported greater incidences of severe dengue and greater mortality. ${ }^{16}$

The available diagnostic assays should be interpreted carefully, and false-negative results are common when serological methods are used because seropositivity may be delayed. Hypersensitivity-dependent skin tests, useful for the diagnosis of latent TB infection (LTBI) (Mantoux reaction or tuberculin 
skin test [TST]) and leishmaniasis (Montenegro test), are Tcell-dependent reactions and frequently reveal falsenegative results. Therefore, interferon-gamma (IFN- $\gamma$ ) release assays (IGRAs)-blood tests that measure T-cell release of IFN- $\gamma$ after stimulation with antigens - have emerged as an interesting alternative. They are also available for LTBI diagnosis and may eventually be promising in other diseases in which the immune memory is reached by an antigen reexposure. ${ }^{17}$ Last, for high-risk patients without a conclusive diagnosis, empirical treatment is often justified, especially in endemic countries, as well as when severe forms are present. ${ }^{18,19}$

Renal allograft dysfunction is commonly detected upon diagnosis or throughout the treatment, with the main causes being hypovolemia, dehydration, sepsis, iodate contrast nephrotoxicity, and drug nephrotoxicity, with amphotericin B being a typical example. ${ }^{20}$ Some drugs, such as azole derivatives used to treat fungal infection (fluconazole, itraconazole, and voriconazole), inhibit P450 cytochrome enzyme activity, leading to a greater exposure to calcineurin inhibitors (CNIs) and enhancing its nephrotoxicity. In contrast, rifampicin, used to treat TB, significantly increases P450 cytochrome activity, reduces $\mathrm{CNI}$ exposure, and favors rejection episodes. Drugs that inhibit or enhance P450 cytochrome activity may also influence exposure to steroids and the mammalian target of rapamycin inhibitors, resulting in toxicity or acute/chronic rejection.

Other causes of allograft dysfunction include the following: local inflammatory and fibrotic activities caused directly by the infectious agent, as described in renal TB and schistosomiasis; rhabdomyolysis in leptospirosis; acute interstitial nephritis in leptospirosis and leishmaniasis; and glomerulonephritis secondary to schistosomiasis, leishmaniasis, and malaria. ${ }^{18,20,21}$

\section{PECULIARITIES OF THE MOST FREQUENT TROPICAL INFECTIONS IN KIDNEY TRANSPLANT PATIENTS}

In this section, we present the peculiarities of the most frequently reported tropical infections in KT patients in Latin America: TB, Chagas disease, leishmaniasis, arboviral diseases, and strongyloidiasis. Other relevant tropical infections, such as malaria, schistosomiasis, and leptospirosis, despite being endemic in this region, have rarely been described in KT patients and are not addressed here. Table 1 summarizes the central information discussed here.

Tuberculosis. The risk of TB infection after organ transplantation is 20 to 50 times greater than that in the general population, even in developing countries. ${ }^{9}$ In Brazil, an endemic area, the incidence of TB is 31 cases per 100,000 people in the general population and $1 \%$ to $5 \%$ in KT recipients. ${ }^{19,22}$ The three pathways of transmission have been reported; reactivation of LTBI is the most common, followed by community acquisition and, more rarely, donor transmission. ${ }^{23}$

As in the general population, pulmonary forms are predominant, regardless of the region's endemicity. However, extrapulmonary and disseminated diseases are more common in KT patients than in immunocompetent individuals. Patients often present with atypical clinical symptoms, even those with typical pulmonary involvement. ${ }^{18,19,24}$ Not rarely, fever of unknown origin is the only symptom, and a high clinical suspicion is necessary, mainly in patients living in endemic areas. ${ }^{18,19}$ Treatment of active TB is based on recommendations for the general population and includes isoniazid, rifampicin, pyrazinamide, and ethambutol. Regarding the main cautions in this population, it is often necessary to adjust the drug dosage for renal function, as hepatotoxicity is more common, ${ }^{25,26}$ and CNI exposure should be monitored closely. Changing rifampicin for rifabutin (a weaker inducer of cytochrome P450) has been suggested for patients with localized and non-severe forms of TB. ${ }^{27}$ Some authors have suggested that rifabutin could be an alternative to replace rifampicin in patients with more severe disease. ${ }^{28}$

Latent TB is the prolonged suppressed state of infection that results from a persistent immune response caused by Mycobacterium tuberculosis antigens, in the absence of clinical signs/symptoms. ${ }^{29}$ Chronic kidney disease patients with $\mathrm{LTBI}$ are at a greater risk for developing active TB after KT when they are administered immunosuppressive drugs. ${ }^{29-31}$ Active TB is characterized by the presence of signs/symptoms and can affect graft and patient outcomes negatively, including a greater risk for kidney dysfunction and mortality. ${ }^{18,29,30}$ Therefore, efforts should be made to prevent TB. In highendemicity areas, clinical suspicion should be high, and pretransplant donor and recipient evaluation should be performed carefully.

All KT candidates should be screened for active TB by careful evaluation of clinical signs and symptoms. Screening for $\mathrm{LTBI}$ is also recommended and involves investigating the history of previously untreated or suspected TB, and near contact with patients with active infection, beyond TST or IGRAs and radiological chest exams. ${ }^{27,31,32}$ The cutoff for TST in this population is not clearly established, and induration of $\geq 5 \mathrm{~mm}$ has been suggested as positive. ${ }^{27}$ Given the anergy of cellular immune response in patients with chronic kidney disease, a tuberculin test may reveal false-negative results. An IFN- $\gamma$ release assay has emerged as an additional tool for improving screening sensitivity in this situation; however, the negative predictive value of IGRAs in high-risk patients is a concern, and pretest probability, including epidemiology and radiological findings, might be considered. Patients living in highendemicity regions should undergo both the TST and IGRAs to maximize the sensitivity of detection. ${ }^{33-35}$

The following options are recommended for LTBI treatment $^{30,34}$ : 6 or 9 months of isoniazid, a 3-month regimen of weekly rifapentine plus isoniazid, 3 to 4 months of isoniazid plus rifampicin, or 3 to 4 months of rifampicin alone. When possible, LTBI treatment should be started before KT.

Graft transmission can be prevented through the screening of active or latent disease in donors, which is only possible in living donations. Active TB in living or deceased donor candidates contraindicates donation. ${ }^{34,35}$ Despite the lack of strong evidence, recipients of allografts from donors with untreated LTBI can be considered for preventive isoniazid chemoprophylaxis. ${ }^{36,37}$ LTBI treatment in potential living donors prior to donation is not clear; however, this might be considered for those living in high-endemicity countries. ${ }^{34-37}$

Chagas disease. Chagas disease is one of the most severe protozoan infections after solid organ transplants-mainly, heart transplantation. ${ }^{13,38}$ The natural history involves an acute and a chronic phase. Acute infection occurs after an incubation period of 1 to 2 weeks following Trypanosoma cruzi transmission. Despite high parasitemia, the acute phase is mostly asymptomatic, mainly in immunocompetent patients, whose immune system controls parasitemia after a few weeks. After the acute phase, most patients progress to the chronic phase, characterized by low-level, intermittent 


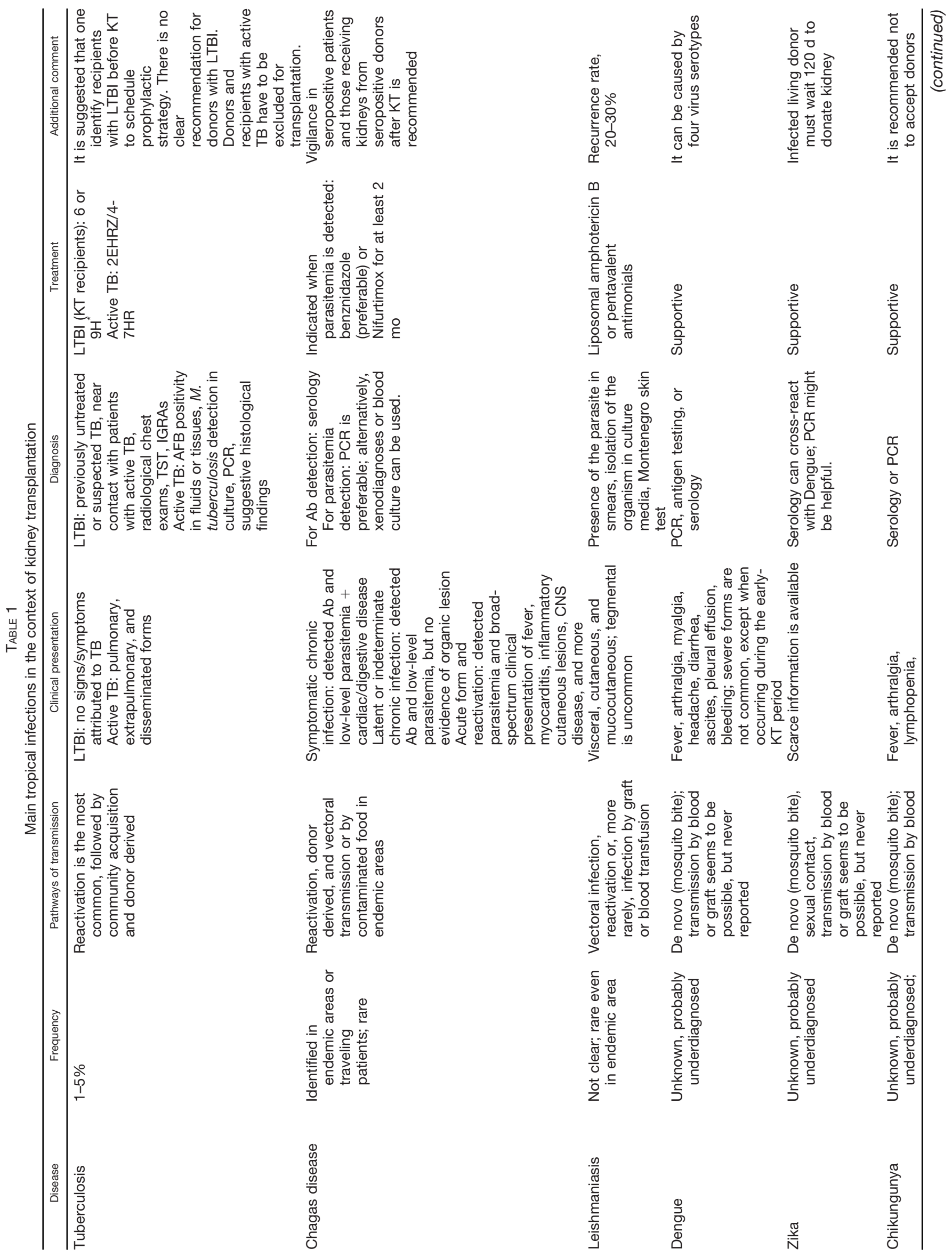




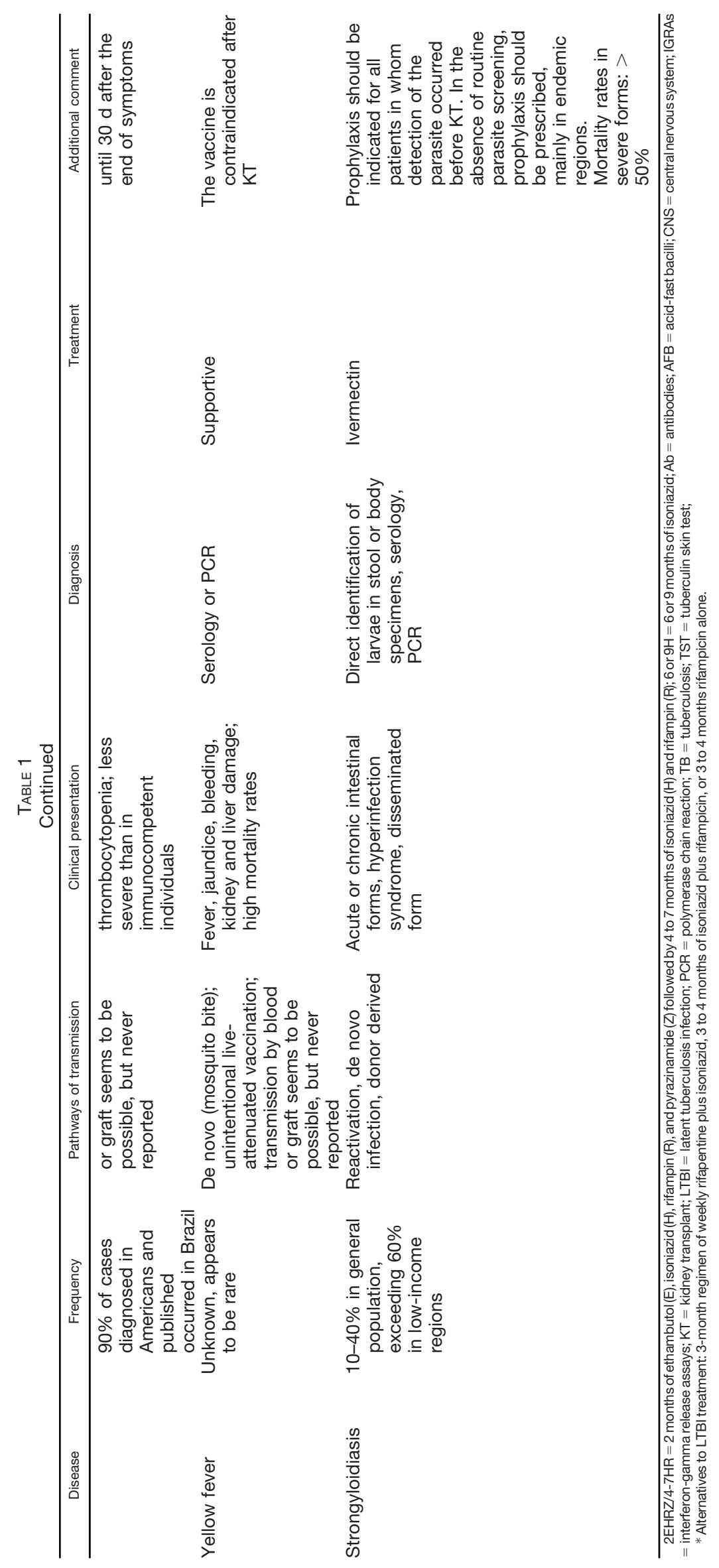


parasitemia. Notwithstanding maintaining positive serology assays, most chronically infected patients do not develop clinical symptoms, known as "indeterminate form" or "latent infection." The rest of the patients who progress to the chronic form present organ damage, mainly with cardiac or gastrointestinal diseases. ${ }^{38,39}$

Three transmission pathways have been described. De novo $T$. cruzi is acquired by a bite from kissing bugs, insects from the Triatominae subfamily, transfusion of contaminated blood, or consumption of infected food, such as an açaí pulp, an Amazonian fruit. ${ }^{39,40}$ The most common mode of infection after $\mathrm{KT}$ is reactivation, which can occur in $8 \%$ to $22 \%$ of seropositive recipients. ${ }^{41,42}$

To avoid donor-derived infection, serological tests should be performed in all potential donors, mainly those living in countries where the disease is endemic. There is no consensus about routine serology performed in donors living in non-endemic regions. In the United States, for instance, less than $20 \%$ of donors in the last decade were screened for Chagas disease. $^{43}$

The risk of untreated KT recipients acquiring Chagas disease from seropositive donors might be considered. Untreated acute Chagas disease in KT recipients is associated with severe manifestations and high mortality rates. In some endemic regions, seropositive patients are not considered for organ donation. ${ }^{39,44}$ However, evidence suggests that when donor-transmitted Chagas disease is diagnosed and treated promptly, it is associated with acceptable outcomes. ${ }^{13,38,44}$

In regions where KT with seropositive donors are allowed, strict laboratory monitoring and clinical surveillance should be adopted, and polymerase chain reaction (PCR) is the method of choice. Blood culture and xenodiagnoses are less sensitive and have a long turnaround time, hampering the timely detection of parasitemia. ${ }^{41,42,45}$ In settings where PCR is not available, surveillance should rely on the direct identification of the parasites in blood using blood smears or, preferably, microhematocrit or the Strout test. A suggested monitoring schedule, based on heart transplantation, is as follows: weekly in the first 2 months, biweekly between the third and sixth months, monthly up to year 2 , and any period after immunosuppression intensification. ${ }^{38}$ Preemptive treatment is only indicated if parasitic load is detected and there is no default threshold to start the treatment in asymptomatic recipients. Treatment is based on benznidazole (first option, better tolerability) or nifurtimox for at least 2 months. ${ }^{38}$ Both drugs can interact with $\mathrm{CNI}$, and immunosuppressive blood levels should be monitored closely. Regardless of whether reactivation occurs, tests should be performed weekly while on treatment until two negative results are obtained. ${ }^{38}$ There are few reports describing the use of routine benznidazole prophylaxis for KT recipients of seropositive donors without parasitemia monitoring. ${ }^{46}$

Chronically infected recipients should be considered at high risk for reactivation, mainly in the first year after $\mathrm{KT}$, and should be monitored closely. ${ }^{38}$ This risk after KT is less than that after heart transplantation; however, it must be considered. Monitoring and management of parasitemia is similar to that described earlier for KT using seropositive donors. ${ }^{47}$

Leishmaniasis. Cellular immune response is critical in Leishmania infections. Immunocompetent patients who acquire this protozoan commonly remain asymptomatic. In contrast, immunosuppressed patients usually present symptomatic forms, including visceral ("kala-azar"), the most frequently described clinical presentation; cutaneous; and mucocutaneous. ${ }^{48,49}$ The real incidence is not clear because it is an uncommon disease in areas with high transplantation activity. Even in endemic countries, reports are limited to single-center case series and case-control analysis. ${ }^{14,50}$ Pathways of transmission may involve acquisition by a vector, as in the general population; reactivation of latent infection; or, more rarely, transfusion with infected blood and donor derived. ${ }^{48}$ If there is no evidence of an active infection, a careful epidemiological and clinical investigation must be performed in donors from endemic regions.

Patients with a previous clinical report of leishmaniasis should be monitored closely, because $20 \%$ to $30 \%$ of patients present with a recurrence after $\mathrm{KT}^{48}$ Secondary prophylaxis with a weekly dose of amphotericin $\mathrm{B}$, monthly dose of antimoniate, or daily dose of fluconazole can be considered, especially in the first year after $\mathrm{KT}^{51}$ For patients traveling to endemic areas, using chemical or physical barriers for protection, such as mosquito repellents and special stockings, is recommended.

Some assays can be considered for diagnosis, depending on staff expertise and local availability, and include the Montenegro test, serology, direct examination of blood samples or bone marrow, or, more currently, PCR. ${ }^{52}$ Because the whole blood cells from patients with active visceral leishmaniasis secrete significant levels of IFN- $\gamma$ and interleukin-10 when stimulated by soluble and specific antigens, IGRAs have emerged as a potential alternative, providing high specificity, but they are not yet used in routine clinical practice. ${ }^{53}$ Liposomal amphotericin B is considered as the first-line treatment, and relapses are frequent. ${ }^{54}$

Arboviral diseases. The most endemic arboviruses in tropical and subtropical areas are dengue, chikungunya, Zika, and yellow fever (YF), all of which have already been reported in KT recipients. ${ }^{55-59}$ Dengue, chikungunya, and Zika transmissions occur through a bite from Aedes aegypti or Aedes albopictus mosquito or, more rarely, through blood transfusion and grafts. Zika can also be transmitted through sexual contact and perinatally. ${ }^{60}$

Dengue can be caused by four virus serotypes, and infection by one serotype does not provide immunity against others. There are a lot of reports and case series on dengue after KT, and it is likely that this infection is underdiagnosed because most cases are oligo- or asymptomatic. ${ }^{61,62}$ Symptomatic infection ranges from mild flu-like symptoms to infection with warming signs, such as abdominal pain, persistent vomiting, mucosal bleed, and lethargy; severe dengue is characterized primarily by plasma leakage with or without hemorrhage or severe organ impairment. ${ }^{9}$ Some evidence has suggested that KT patients are less likely to develop severe dengue. ${ }^{9}$ However, a recently published systematic review reported that pleural effusions and ascites were more frequent in that population, and a greater incidence of severe dengue and higher mortality rate was observed. ${ }^{16}$ Two cases of severe dengue were described in the early postoperative period, suggesting greater severity when the patient undergoes transplantation during the incubation period. ${ }^{63}$ Diagnosis can be confirmed by antigen testing, PCR, or serology depending on the time of symptoms; antigen testing and PCR are used in the early stages and serology in the later stages of the infection. ${ }^{64}$ Treatment is limited to supportive care. 
Zika infection may result in neonatal microcephaly, neurological complications, and Guillain-Barré syndrome in the general population. ${ }^{65}$ Diagnosis can be achieved via serology, and treatment is restricted to supportive measures. Thus far, there are only a few reported cases of Zika after KT, both occurring during the late post-transplant period and with favorable outcomes. ${ }^{66}$ Living donor candidates with a history of Zika infection should wait at least 120 days after infection resolution to donate. $^{67}$

Currently, Brazil accounts for more than $90 \%$ of confirmed cases of chikungunya fever in the Americas. ${ }^{68}$ After a mosquito bite, viremia can extend up to 3 weeks. ${ }^{55}$ Recent reports have shown that patients receiving steroid therapy usually exhibit less severe disease; to date, only a few case reports and case series on chikungunya after KT have been published. ${ }^{69-72}$ Fever, arthralgia, lymphopenia, and thrombocytopenia are the most common manifestations. ${ }^{68}$ Arthralgia, often affecting large and symmetric joints, is milder and has a shorter duration compared with immunocompetent individuals ${ }^{71,73,74}$; no negative impact on graft outcomes has been described. ${ }^{71}$

The diagnosis can be confirmed through serology (IgM), but PCR is the gold standard. Treatment is usually restricted to symptom-based management. ${ }^{75}$ It is recommended not to accept donors until 30 days after the symptoms end. ${ }^{76}$

YF is considered one of the most severe arboviral infections and is associated with high mortality rates, independent of the baseline patient characteristics. There are two epidemiological cycles of YF disease: wild and urban. Vectorial transmission of the wild form occurs through the bite of Sabethes and Haemagogus mosquitoes. The urban form is transmitted by Aedes aegypti. Approximately $90 \%$ of all cases are in Africa, where both forms have been identified; the urban form is considered to have been eradicated in America. ${ }^{56}$

In the elderly, children, patients with organ impairment (heart, lung, liver, or kidney), and those undergoing immunosuppressive therapy usually have worse prognosis for YF. ${ }^{59}$ In the general population, peak viremia occurs 3 days after transmission, and after 10 days on average, patients present with fever, jaundice, hemorrhages, and kidney and liver damage. ${ }^{56,59}$ In a recent Brazilian YF outbreak, a myriad of organ and tissue disorders were observed, including fulminant hepatitis, coagulation disorders and bleeding, acute kidney injury, systemic vasculopathy, pancreatitis, intestinal ischemia, splenitis, hemophagocytosis, and immune dysfunction. ${ }^{77}$ Two regions reported their experience with liver transplantation to manage fulminant hepatitis. ${ }^{77,78}$ Because there are only a few reports on YF after $\mathrm{KT}$, there is no robust information on the clinical presentation and prognosis in this population. To date, no blood or graft transmission has been reported in this population. ${ }^{58,59}$ Treatment is based on supportive measures. The available vaccine is live attenuated and, therefore, contraindicated in immunocompromised patients. ${ }^{79}$

Strongyloidiasis. Infection by Strongyloides stercoralis is endemic in some tropical regions, although its real prevalence has varied greatly among different countries. The global prevalence is heterogeneous, and evidence suggests that it can affect $10 \%$ to $40 \%$ of the population in many tropical and subtropical countries, exceeding $60 \%$ in low-income countries, where ecological, sanitary, and social situations are fragile. ${ }^{80}$

Reactivation is the most frequent pathway of transmission, but de novo acquisition and transmission by grafts have also been reported. ${ }^{81,82}$ Clinically, these infections are classified into four forms: acute intestinal form, chronic intestinal form, hyperinfection syndrome, and disseminated strongyloidiasis. ${ }^{83}$ The acute intestinal presentation is characterized by nonspecific abdominal pain, followed by symptoms in the upper or lower gastrointestinal tract such as nausea, vomiting, or diarrhea, and is associated sporadically with cutaneous lesions and lung symptoms (Löffler syndrome). The chronic intestinal form is more frequent and is usually confounded by functional dyspepsia or irritable bowel syndrome. Few patients may progress to severe presentations, followed by paralytic ileus, intestinal obstruction, or gastrointestinal bleeding. These severe intestinal cases associated with severe lung manifestations such as dyspnea and hemoptysis are known as hyperinfection syndromes, which involve amplification of the natural cycle of the parasite and are more common in immunosuppressed patients. In these three forms (acute and chronic intestinal forms and hyperinfection syndrome), larvae are only found in organs involved in the infection cycle. Last, in disseminated strongyloidiasis, patients commonly present with systemic complications such as meningitis, cholecystitis, liver abscess, pancreatitis, or septic shock; thus, larvae are found at sites different from those during their natural cycle. $^{84}$

Severe forms are associated with mortality rates reaching $50 \%$, especially during the early post-KT period or after acute rejection treatment, when high-exposure immunosuppression is used. $^{85}$ A recent multicenter Brazilian study reported a 30 -day all-cause mortality rate of $28.3 \%$, and Gramnegative bacteremia and respiratory failure were predictors of mortality. ${ }^{15}$

The diagnosis is based primarily on the isolation of $S$. stercoralis from biological samples: stool, duodenum aspirate, and other body fluids. ${ }^{86}$ Because the sensitivity of this method is not ideal, serological assays and molecular biology techniques (PCR) have been suggested as alternatives or additional tools. ${ }^{87,88}$ Treatment is preferably accomplished with ivermectin for 2 days, and a second course can be indicated 1 or 2 weeks later. In patients with severe forms, treatment should be continued until symptoms improve and serial stool samples are negative for 2 weeks. ${ }^{84}$

Routinely, Strongyloides screening and treatment should be considered as a strategy to prevent both recipient- and donorderived strongyloidiasis. Available screening strategies are the parasitological stool sample exam and serology, both with limited accuracy. ${ }^{89,90}$ Preemptive treatment should be instituted for all KT recipients with positive serology or larvae in the stools assessed prior to KT. In endemic countries or in patients transplanted with unknown Strongyloides status, universal prophylaxis might be considered. Treatment is preferably indicated with ivermectin for 2 days 2 weeks prior to KT in cases of scheduled transplants (living donor transplants), or shortly after surgery in nonscheduled KT (deceased donor transplants). It is also indicated after treatment of acute rejection or when intensification of immunosuppression for any other reason is required. ${ }^{84}$

\section{GENERAL ASPECTS OF PREVENTION AND OTHER SPECIFIC SITUATIONS}

Strategies for the prevention of infectious diseases in KT patients can be divided into three categories: 1) prevention of donor-derived transmission, 2) prevention of post- 
transplant reactivation of latent infections, and 3) prevention of infections acquired in the community. During the pretransplant clinical evaluation, two strategies should be used: screening for selected infectious diseases and updating the vaccination schedule. The immunization schedule must be updated in all KT candidates according to local recommendations. There is no robust information on the safety of YF immunization in patients undergoing dialysis or on a waiting list. $A$ single-center Brazilian study reported only minor adverse events in $24 \%$ of 45 patients undergoing chronic dialysis who received the YF vaccine, suggesting that it is likely the vaccine is safe in this population. ${ }^{91}$ Thus, KT candidates should receive the YF vaccine before transplantation and wait for at least 8 weeks before undergoing transplantation.

It is important to mention infection by HTLV-1, which can cause tropical spastic paralysis, neurogenic bladder, and T-cell lymphoma, and that transmission via the graft can be expected. In some countries, HTLV-1 serology for kidney donors is no longer recommended because it is often false positive, and the probability of unexpected donor-derived transmission is low. However, donor-derived HTLV-1 has already been described and can be associated with significant morbidity. ${ }^{92,93}$ Therefore, HTLV-1-positive serology must be verified using more specific methods such as Western blot or PCR; and, if infection is confirmed, potential donors must be excluded. There is no standardized recommendation for follow-up in seropositive recipients or for patients infected inadvertently by graft transmission. ${ }^{94}$

Regarding malaria, candidates for donation with active infection must be excluded, and in suspected cases, diagnostic confirmation via blood smear or PCR testing is recommended. Patients traveling to endemic areas should be advised of prophylactic environmental exposure measures, and, in some cases, chemoprophylaxis with doxycycline, chloroquine, mefloquine, or atovaquone may be indicated, and patients should be monitored closely for the potential interaction between these drugs and immunosuppressive agents. $^{95}$

There are no specific prophylactic recommendations to prevent dengue, Zika, and chikungunya fever. People who live in endemic areas or travel to these areas must be strongly encouraged to use mosquito repellents and physical barriers.

\section{FUTURE PERSPECTIVES AND CONCLUSIONS}

Tropical infections in KT patients have begun to receive great interest, mainly in regions such as Latin America, where transplant programs have improved. In contrast to the typical diseases that usually affect this specific population, tropical infections are more frequent in countries where there are particularly weak points in the public health system. Therefore, worldwide efforts to share advancement in strategies for case notification and, more importantly, for diagnosis are necessary. Furthermore, actions focused on prophylaxis must be improved and expanded, including the development of effective and safe vaccines, chemoprophylaxis when indicated, and public information. Considerable attention must be given to the anti-vaccination movement, which is a harmful new movement responsible for the decline in the number of vaccinated people worldwide. Last, further research aimed at developing new vaccines, new prophylaxis strategies, and more sensitive diagnostic techniques should be encouraged in rich and poor countries alike.

Received December 13, 2019. Accepted for publication April 12, 2021. Published online June 28, 2021.

Financial support: This work was supported by the Programa de Apoio ao Desenvolvimento Institucional do Sistema Único de SaúdePROADI-SUS program (Brazilian Health Ministry) and the National Council for Scientific and Technological Development (Conselho Nacional de Desenvolvimento Científico e Tecnológico).

Authors' addresses: Lúcio R. Requião-Moura, Renal Transplant Unit, Hospital Israelita Albert Einstein, São Paulo, Brazil, and Nephrology Division, Federal University of São Paulo, São Paulo, Brazil, E-mail: lucio. moura@einstein.br. Elizabeth De Francesco Daher, Savio de Oliveira Brilhante, Silvana Daher Costa, and Tainá Veras de Sandes-Freitas, Department of Clinical Medicine, Federal University of Ceará, Fortaleza, Brazil, E-mails: ef.daher@yahoo.com.br, saviobrilhante2009@hotmail. com, silvanadaher1@hotmail.com, and taina.sandes@gmail.com. Cassio R. Moreira Albino, Renal Transplant Unit, Hospital Israelita Albert Einstein, São Paulo, Brazil, E-mail: kssio_rafael@hotmail.com. Geraldo Bezerra da Silva Jr., Medical School, University of Fortaleza, Fortaleza, Ceará, Brazil, E-mail: geraldobezerrajr@unifor.br.

\section{REFERENCES}

1. Cusumano AM, Di Gioia C, Hermida O, Lavorato C, 2005. Latin American Registry of Dialysis and Renal Transplantation: the Latin American Dialysis and Renal Transplantation Registry Annual Report 2002. Kidney Int Suppl 97: S46-S52.

2. de Castro Rodrigues Ferreira F, Cristelli MP, Paula MI, Proença H, Felipe CR, Tedesco-Silva H, Medina-Pestana JO, 2017. Infectious complications as the leading cause of death after kidney transplantation: analysis of more than 10,000 transplants from a single center. $J$ Nephrol 30: 601-606.

3. Bicalho PR, Requião-Moura LR, Arruda ÉF, Chinen R, Mello L, Bertocchi APF, Lamkowski Naka E, Tonato EJ, Pacheco-Silva A, 2019. Long-term outcomes among kidney transplant recipients and after graft failure: a single-center cohort study in Brazil. BioMed Res Int 2019: 7105084.

4. El-Zoghby ZM, Stegall MD, Lager DJ, Kremers WK, Amer H, Gloor JM, Cosio FG, 2009. Identifying specific causes of kidney allograft loss. Am J Transplant 9: 527-535.

5. Franco-Paredes C, Jacob JT, Hidron A, Rodriguez-Morales AJ, Kuhar D, Caliendo AM, 2010. Transplantation and tropical infectious diseases. Int J Infect Dis 14: e189-e196.

6. Martín-Dávila $P$ et al., 2008. Transmission of tropical and geographically restricted infections during solid-organ transplantation. Clin Microbiol Rev 21: 60-96.

7. Valar $C$ et al., 2007. Parasitic infection in renal transplant recipients. Transplant Proc 39: 460-462.

8. Fishman JA, 2017. Infection in organ transplantation. Am J Transplant 17: 856-879.

9. Machado CM, Martins TC, Colturato I, Leite MS, Simione AJ, de Souza MP, Mauad MA, Colturato VR, 2009. Epidemiology of neglected tropical diseases in transplant recipients: review of the literature and experience of a Brazilian HSCT center. Rev Inst Med Trop São Paulo 51: 309-324.

10. Sawyer RG, Crabtree TD, Gleason TG, Antevil JL, Pruett TL, 1999. Impact of solid organ transplantation and immunosuppression on fever, leukocytosis, and physiologic response during bacterial and fungal infections. Clin Transplant 13: 260-265.

11. Mahmoud KM, Sobh MA, El-Agroudy AE, Mostafa FE, Baz ME, Shokeir AA, Ghoneim MA, 2001. Impact of schistosomiasis on patient and graft outcome after renal transplantation: 10 years' follow-up. Nephrol Dial Transplant 16: 2214-2221.

12. Barsoum RS, 2006. Parasitic infections in transplant recipients. Nat Clin Pract Nephrol 2: 490-503.

13. Kotton CN, Lattes R, AST Infectious Diseases Community of Practice, 2009. Parasitic infections in solid organ transplant recipients. Am J Transplant 9 (Suppl 4): S234-S251. 
14. de Silva AA et al., 2015. Epidemiologic, clinical, diagnostic and therapeutic aspects of visceral leishmaniasis in renal transplant recipients: experience from thirty cases. BMC Infect Dis 15: 96.

15. Miglioli-Galvão L et al., 2020. Severe Strongyloides stercoralis infection in kidney transplant recipients: a multicenter casecontrol study. PLoS Negl Trop Dis 14. doi: 10.1371/ journal.pntd.0007998.

16. Weerakkody RM, Patrick JA, Sheriff MHR, 2017. Dengue fever in renal transplant patients: a systematic review of literature. $B M C$ Nephrol 18. doi: 10.1186/s12882-016-0428-y.

17. Redelman-Sidi G, Sepkowitz KA, 2013. IFN- $\gamma$ release assays in the diagnosis of latent tuberculosis infection among immunocompromised adults. Am J Respir Crit Care Med 188: 422-431.

18. Costa SD, de Sandes-Freitas TV, Jacinto CN, Martiniano LVM, Amaral YS, Paes FJVN, Sales ML de MBO, Esmeraldo $\mathrm{R}$ de $\mathrm{M}$, Daher E de F, 2017. Tuberculosis after kidney transplantation is associated with significantly impaired allograft function. Transpl Infect Dis 19. doi: 10.1111/tid.12750.

19. Viana LA, Cristelli MP, Santos DW, Tavares MG, Dantas MTC, Felipe CR, Silva HT, Pestana JM, 2019. Influence of epidemiology, immunosuppressive regimens, clinical presentation, and treatment on kidney transplant outcomes of patients diagnosed with tuberculosis: a retrospective cohort analysis. Am J Transplant 19: 1421-1431.

20. Daher EDF, da Junior Silva GB, Vieira APF, de Souza JB, Falcão FDS, da Costa CR, Fernandes AAC da S, Lima RSA, 2014. Acute kidney injury in a tropical country: a cohort study of 253 patients in an infectious diseases intensive care unit. Rev Soc Bras Med Trop 47: 86-89.

21. Daher EDF, de Abreu KLS, da Silva GB Junior, 2010. Leptospirosis-associated acute kidney injury. J Bras Nefrol 32: 400-407.

22. Meinerz G, da Silva CK, Goldani JC, Garcia VD, Keitel E, 2016. Epidemiology of tuberculosis after kidney transplantation in a developing country. Transpl Infect Dis 18: 176-182.

23. Marques IDB et al., 2013. Clinical features and outcomes of tuberculosis in kidney transplant recipients in Brazil: a report of the last decade. Clin Transplant 27: E169-E176.

24. Gras J et al., 2018. Clinical characteristics, risk factors, and outcome of tuberculosis in kidney transplant recipients: a multicentric case-control study in a low-endemic area. Transpl Infect Dis 20: e12943.

25. Chen $\mathrm{C}-\mathrm{Y}$ et al., 2015. Incidence and risk factors for tuberculosis after liver transplantation in an endemic area: a nationwide population-based matched cohort study. Am J Transplant 15: 2180-2187.

26. Higuita LMS et al., 2014. Tuberculosis in renal transplant patients: the experience of a single center in Medellín-Colombia, 2005-2013. Braz J Nephrol 36: 512-518.

27. Aguado JM, Torre-Cisneros J, Fortún J, Benito N, Meije Y, Doblas A, Muñoz P, 2009. Tuberculosis in solid-organ transplant recipients: consensus statement of the Group for the Study of Infection in Transplant Recipients (GESITRA) of the Spanish Society of Infectious Diseases and Clinical Microbiology. Clin Infect Dis 48: $1276-1284$.

28. Meije Y, Piersimoni C, Torre-Cisneros J, Dilektasli AG, Aguado JM, 2014. Mycobacterial infections in solid organ transplant recipients. Clin Microbiol Infect 20: 89-101.

29. Mack $U$ et al., 2009. LTBI: latent tuberculosis infection or lasting immune responses to $M$. tuberculosis? A TBNET consensus statement. Eur Respir J 33: 956-973.

30. Dye C, Scheele S, Dolin P, Pathania V, Raviglione MC, 1999. Global burden of tuberculosis: estimated incidence, prevalence, and mortality by country. JAMA 282: 677-686.

31. Krishnamoorthy S, Kumaresan N, Zumla A, 2019. Latent tuberculosis infection and renal transplantation: diagnosis and management. Int $J$ Infect Dis 80: S73-S76.

32. WHO, 2018. Guidelines on the Management of Latent Tuberculosis Infection. Available at: http://www.who.int/tb/publications/ latent-tuberculosis-infection/en/. Accessed May 16, 2021.

33. Muñoz L, Santin M, 2016. Prevention and management of tuberculosis in transplant recipients: from guidelines to clinical practice. Transplantation 100: 1840-1852.

34. Santoro-Lopes G, Subramanian AK, Molina I, Aguado JM, Rabagliatti $R$, Len $O, 2018$. Tuberculosis recommendations for solid organ transplant recipients and donors. Transplantation 102 (Suppl 2): S60-S65.

35. Subramanian AK, Morris MI, 2013. Mycobacterium tuberculosis infections in solid organ transplantation. Am J Transplant 13: 68-76.

36. Bumbacea D et al., 2012. The risk of tuberculosis in transplant candidates and recipients: a TBNET consensus statement. Eur Respir J 40: 990-1013.

37. Morris Ml et al., 2012. Diagnosis and management of tuberculosis in transplant donors: a donor-derived infections consensus conference report. Am J Transplant 12: 2288-2300.

38. Pierrotti LC, Carvalho NB, Amorin JP, Pascual J, Kotton CN, López-Vélez R, 2018. Chagas disease recommendations for solid-organ transplant recipients and donors. Transplantation 102 (Suppl 2): S1-S7.

39. Bern C, 2015. Chagas' disease. N Engl J Med 373: 1882.

40. de Souza-Lima R de $\mathrm{C}$ et al., 2013. Outbreak of acute Chagas disease associated with oral transmission in the Rio Negro region, Brazilian Amazon. Rev Soc Bras Med Trop 46: 510-514.

41. Riarte A et al., 1999. Chagas' disease in patients with kidney transplants: 7 years of experience 1989-1996. Clin Infect Dis 29: 561-567.

42. Lattes R, Lasala MB, 2014. Chagas disease in the immunosuppressed patient. Clin Microbiol Infect 20: 300-309.

43. Schwartz BS, Paster M, Ison MG, Chin-Hong PV, 2011. Organ donor screening practices for Trypanosoma cruzi infection among US organ procurement organizations. Am J Transplant 11: 848-851.

44. Silva AE et al., 2010. Acute Chagas' disease in postrenal transplant and treatment with benznidazole. Ann Diagn Pathol 14: 199-203.

45. Huprikar S et al., 2013. Donor-derived Trypanosoma cruzi infection in solid organ recipients in the United States, 2001-2011. Am J Transplant 13: 2418-2425.

46. Sousa AA, Lobo MCSG, Barbosa RA, Bello V, 2004. Chagas seropositive donors in kidney transplantation. Transplant Proc 36: 868-869.

47. Kransdorf EP, Zakowski PC, Kobashigawa JA, 2014. Chagas disease in solid organ and heart transplantation. Curr Opin Infect Dis 27: 418-424.

48. van Griensven J, Carrillo E, López-Vélez R, Lynen L, Moreno J, 2014. Leishmaniasis in immunosuppressed individuals. Clin Microbiol Infect 20: 286-299.

49. Antinori S, Cascio A, Parravicini C, Bianchi R, Corbellino M, 2008. Leishmaniasis among organ transplant recipients. Lancet Infect Dis 8: 191-199.

50. Alves da Silva A, Pacheco-Silva A, de Castro Cintra Sesso R, Esmeraldo RM, Costa de Oliveira CM, Fernandes PFCBC, Oliveira RA, Silva LSV, Carvalho VP, Nery Costa $\mathrm{CH}, 2013$. The risk factors for and effects of visceral leishmaniasis in graft and renal transplant recipients. Transplantation 95: 721-727.

51. Copeland NK, Aronson NE, 2015. Leishmaniasis: treatment updates and clinical practice guidelines review. Curr Opin Infect Dis 28: 426-437: 1-6.

52. Carrillo E, Carrasco-Antón N, López-Medrano F, Salto E, Fernández L, San Martín JV, Alvar J, Aguado JM, Moreno J, 2015. Cytokine release assays as tests for exposure to leishmania, and for confirming cure from leishmaniasis, in solid organ transplant recipients. PLoS Negl Trop Dis 9: e0004179.

53. Singh OP, Sundar S, 2014. Whole blood assay and visceral leishmaniasis: challenges and promises. Immunobiology 219: 323328.

54. Clemente WT, Mourão PHO, Lopez-Medrano F, Schwartz BS, García-Donoso C, Torre-Cisneros J, 2018. Visceral and cutaneous leishmaniasis recommendations for solid organ transplant recipients and donors. Transplantation 102 (Suppl 2): S8-S15.

55. Darrigo LG, de Sant'Anna Carvalho AM, Machado CM, 2018. Chikungunya, dengue, and Zika in immunocompromised hosts. Curr Infect Dis Rep 20: 5.

56. Barnett ED, 2007. Yellow fever: epidemiology and prevention. Clin Infect Dis 44: 850-856.

57. Lindsey NP, Rabe IB, Miller ER, Fischer M, Staples JE, 2016. Adverse event reports following yellow fever vaccination, 2007-13. J Travel Med 23. doi: 10.1093/jtm/taw045. 
58. de Sousa MV, Zollner R de L, Stucchi RSB, Boin I de FSF, de Ataide EC, Mazzali M, 2019. Yellow fever disease in a renal transplant recipient: case report and literature review. Transpl Infect Dis 21: e13151.

59. Pierrotti LC, Duarte-Neto AN, Song ATW, Ventura CG, David-Neto E, Sergio de Azevedo L, 2019. Fatal yellow fever in a kidney transplant patient. Clin Infect Dis 70: 144-148. doi: 10.1093/ $\mathrm{cid} / \mathrm{ciz} 389$.

60. Motta IJF et al., 2016. Evidence for transmission of Zika virus by platelet transfusion. N Engl J Med 375: 1101-1103.

61. Nasim A, Anis S, Baqi S, Akhtar SF, Baig-Ansari N, 2013. Clinical presentation and outcome of dengue viral infection in liverelated renal transplant recipients in Karachi, Pakistan. Transpl Infect Dis 15: 516-525.

62. Fernandes PFCBC et al., 2017. Dengue in renal transplant recipients: clinical course and impact on renal function. World $J$ Transplant 7: 57-63.

63. Maia SHF, Brasil IRC, Esmeraldo RDM, Ponte CND, Costa RCS, Lira RA, 2015. Severe dengue in the early postoperative period after kidney transplantation: two case reports from Hospital Geral de Fortaleza. Rev Soc Bras Med Trop 48: 783-785.

64. Tang KF, Ooi EE, 2012. Diagnosis of dengue: an update. Expert Rev Anti Infect Ther 10: 895-907.

65. Mlakar $\mathrm{J}$ et al., 2016. Zika virus associated with microcephaly. $N$ Engl J Med 374: 951-958.

66. Nogueira ML, Estofolete CF, Terzian ACB, Mascarin do Vale EPB, da Silva RCMA, da Silva RF, Ramalho HJ, Fernandes Charpiot IMM, Vasilakis N, Abbud-Filho M, 2017. Zika virus infection and solid organ transplantation: a new challenge. Am J Transplant 17: 791-795.

67. Moura-Neto JA, Braga Silva CA, Moura AF, Rocco Suassuna JH, 2019. Emergent arboviruses and renal transplantation: a global challenge. Kidney Int Rep 4: 647-655.

68. Collucci C, 2016. Brazil sees sharp rise in chikungunya cases. BMJ 354: 14560 .

69. Gasperina DD, Balsamo ML, Garavaglia SD, Rovida F, Baldanti F, Grossi PA, 2015. Chikungunya infection in a human immunodeficiency virus-infected kidney transplant recipient returning to Italy from the Dominican Republic. Transpl Infect Dis 17: 876879 .

70. Girão ES et al., 2017. Chikungunya infection in solid organ transplant recipients. Transplant Proc 49: 2076-2081.

71. Pierrotti LC, Lopes MIBF, do Nascimento AP, Caiaffa-Filho H, Lemos FBC, Reusing JO, Sejas ONE, David-Neto E, Azevedo LS, 2017. Chikungunya in kidney transplant recipients: a series of cases. Int J Infect Dis 64: 96-99.

72. Rosso F, Rodríguez S, Cedano JA, Mora BL, Moncada PA, Velez JD, 2018. Chikungunya in solid organ transplant recipients: a case series and literature review. Transpl Infect Dis 20: e12978.

73. Staikowsky F, Talarmin F, Grivard P, Souab A, Schuffenecker I, Le Roux K, Lecuit M, Michault A, 2009. Prospective study of chikungunya virus acute infection in the Island of La Réunion during the 2005-2006 outbreak. PLoS One 4: e7603.

74. Kee ACL, Yang S, Tambyah P, 2010. Atypical chikungunya virus infections in immunocompromised patients. Emerg Infect Dis 16: $1038-1040$.

75. Delang L et al., 2014. Mutations in the chikungunya virus nonstructural proteins cause resistance to favipiravir (T-705), a broad-spectrum antiviral. J Antimicrob Chemother 69: 27702784.

76. Moura-Neto JA, Moura AF de S, Souza E, Henriques JL de C, Arruda MB, Pessoa LS, Boullosa LT, Junior O da CF, Suassuna JHR, 2017. Successful live kidney transplantation after chikungunya disease in the donor. Kidney Int Rep 2: 1250-1253.
77. Duarte-Neto AN et al., 2019. Yellow fever and orthotopic liver transplantation: new insights from the autopsy room for an old but re-emerging disease. Histopathology 77: 638-648.

78. Vieira $V$ et al., 2019. Liver transplantation for acute liver failure due to yellow fever: a case report. Transplant Proc 51: 1625-1628.

79. Azevedo LS et al., 2012. Yellow fever vaccination in organ transplanted patients: is it safe? A multicenter study. Transpl Infect Dis 14: 237-241

80. Schär F, Trostdorf U, Giardina F, Khieu V, Muth S, Marti H, Vounatsou P, Odermatt P, 2013. Strongyloides stercoralis: global distribution and risk factors. PLoS Negl Trop Dis 7: e2288.

81. Keiser PB, Nutman TB, 2004. Strongyloides stercoralis in the immunocompromised population. Clin Microbiol Rev 17: 208217.

82. Abanyie FA et al., 2015. Donor-derived Strongyloides stercoralis infection in solid organ transplant recipients in the United States, 2009-2013. Am J Transplant 15: 1369-1375.

83. Marcos LA, Terashima A, Canales M, Gotuzzo E, 2011. Update on strongyloidiasis in the immunocompromised host. Curr Infect Dis Rep 13: 35-46.

84. Camargo LFA, Kamar N, Gotuzzo E, Wright AJ, 2018. Schistosomiasis and strongyloidiasis recommendations for solid-organ transplant recipients and donors. Transplantation 102 (Suppl 2): S27-S34.

85. Ferreira CJA, da Silva DA, Almeida PH, da Silva LSV, Carvalho VP, Coutinho AF, Pinheiro FG, Maia RP, da Silva Junior GB, de Oliveira RA, 2012. Fatal disseminated strongyloidiasis after kidney transplantation. Rev Soc Bras Med Trop 45: 652-654.

86. Siddiqui AA, Berk SL, 2001. Diagnosis of Strongyloides stercoralis infection. Clin Infect Dis 33: 1040-1047.

87. Kalantari N, Chehrazi M, Ghaffari S, Gorgani-Firouzjaee T, 2020. Serological assays for the diagnosis of Strongyloides stercoralis infection: a systematic review and meta-analysis of diagnostic test accuracy. Trans R Soc Trop Med Hyg 114: 459-469.

88. Paula FM et al., 2018. Molecular diagnosis of Strongyloides stercoralis among transplant candidates. Transpl Infect Dis 20: e12909.

89. Camargo JF et al., 2019. Implementation of a Strongyloides screening strategy in solid organ transplant donors and recipients. Clin Transplant 33: e13497.

90. Gómez-Junyent J, Paredes D, Hurtado JC, Requena-Méndez A, Ruiz A, Valls ME, Vila J, Muñoz J, 2018. High seroprevalence of Strongyloides stercoralis among individuals from endemic areas considered for solid organ transplant donation: a retrospective serum-bank based study. PLoS Negl Trop Dis 12: e0007010.

91. Facincani T, Guimarães MNC, De Sousa Dos Santos S, 2016. Yellow fever vaccination status and safety in hemodialysis patients. Int J Infect Dis 48: 91-95.

92. Shingde R, Habachou LI, Calisa V, Craig JC, Tong A, Chen SC-A, Wong G, 2018. Unexpected donor-derived infectious transmissions by kidney transplantation: a systematic review. Transpl Infect Dis 20: e12851.

93. de Mendoza C et al., 2019. HTLV-1 infection in solid organ transplant donors and recipients in Spain. BMC Infect Dis 19: 706.

94. Moreno-Ajona D, Yuste JR, Martín P, Gállego Pérez-Larraya J, 2018. HTLV-1 myelopathy after renal transplant and antiviral prophylaxis: the need for screening. J Neurovirol 24: 523-525.

95. Pierrotti LC, Levi ME, Di Santi SM, Segurado AC, Petersen E, 2018. Malaria disease recommendations for solid organ transplant recipients and donors. Transplantation 102 (Suppl 2): S16-S26. 